\title{
Modelo de previsão de insolvência para cooperativas de crédito
}

\author{
Michel Alexandre*
}

RESUMO - O presente trabalho propõe-se a desenvolver um modelo de previsão de insolvência para cooperativas de crédito. Apesar de minoritário dentro do sistema financeiro nacional, o setor cooperativista de crédito brasileiro vem ampliando sua importância relativa e desempenha um papel fundamental no que concerne ao desenvolvimento local. Por estas razões, vê-se como necessário um melhor entendimento dos fatores ligados à insolvência das cooperativas de crédito. $\mathrm{O}$ modelo aqui desenvolvido aponta inadimplência, rentabilidade, alavancagem, capitalização, liquidez e filiação a alguma cooperativa central como alguns dos elementos ligados à fragilização futura das cooperativas de crédito.

Palavras-chave: Sistema financeiro nacional. Cooperativas de crédito. Modelo de previsão de insolvência.

\section{INTRODUÇÃO}

Este trabalho propõe-se a apresentar um modelo de previsão de insolvência para cooperativas de crédito. Esta proposta justifica-se por três razões: i) a crescente importância do sistema cooperativo dentro do sistema financeiro nacional, ii) a relevância das cooperativas para o desenvolvimento local e iii) a escassez de estudos sobre o tema.

O peso das cooperativas de crédito no Brasil ainda é pequeno quando comparado ao de outros países ${ }^{1}$. Em dezembro de 2006, as cooperativas respondiam por apenas 2,26\% das operações de crédito do sistema bancário e atendiam a pouco mais de três milhões de pessoas no Brasil, menos de 2\% da população do país (PINHEIRO, 2008). No entanto, o sistema cooperativista de crédito vem adquirindo crescente importância no país. De acordo com dados do Banco Central, o número de cooperativas saltou de 946 em 1994 para mais de 1.400 em 2006. Além disso, a participação das cooperativas no crédito do sistema bancário era de apenas 0,44\% em 1995, valor bem inferior ao observado em 2006.

Ainda que as cooperativas ocupem uma posição minoritária dentro do sistema financeiro, elas possuem um papel fundamental no que tange ao desenvolvimento local. Isso

\footnotetext{
* Doutorando em Economia, Università di Siena (Itália), e Banco Central do Brasil. Endereço eletrônico: michel.alexandre@bcb.gov.br. As visões expressas neste trabalho não refletem necessariamente a opinião do Banco Central do Brasil. Gostaria de agradecer aos colegas do Departamento de Supervisão de Cooperativas e de Instituições Não Bancárias (DESUC), especialmente Flávio de Melo Pereira, por comentários e sugestões. Erros remanescentes são de minha inteira responsabilidade.

${ }^{1}$ Sobre o peso das cooperativas de crédito em outros países, ver WOCCU (2008).
} 
se dá porque as cooperativas assumem os riscos de suas aplicações em prol da comunidade em que se localizam, promovendo o desenvolvimento local sustentável através da formação de poupança e do microcrédito direcionado a iniciativas empresariais locais (SOARES \& MELO SOBRINHO, 2008).

É importante, portanto, um melhor conhecimento acerca dos elementos relacionados à sobrevivência das cooperativas de crédito. No entanto, estudos sobre o tema ainda são escassos. A maioria dos estudos sobre previsão de falência de instituições financeiras brasileiras é voltada para bancos².

Além desta introdução, este trabalho é composto por mais duas partes. Os dados e a metodologia utilizados no desenvolvimento do modelo de previsão de insolvência, assim como os resultados do mesmo, são apresentados na Seção 2. Comentários finais tomam a última seção.

\section{DADOS E ESTIMAÇÃO DO MODELO}

As informações utilizadas neste trabalho são referentes a dezembro de 2006. As variáveis independentes dividem-se em: i) informações estruturais (Tabela 1) e ii) 23 indicadores econômico-financeiros (Tabela 2). A variável dependente, EVID, é binária e assume o valor igual a um caso a cooperativa tenha sido classificada como "em evidência" entre janeiro/2007 e junho $/ 2009^{3}$. Trabalhou-se com o universo de cooperativas singulares da data-base. Em dezembro de 2006, havia 1.388 cooperativas singulares. Foram excluídas aquelas já em evidência (104) e as que possuíam alguma informação faltante (95). Ao final, trabalhou-se com 1.189 cooperativas.

A estimação do modelo seguiu duas etapas. Primeiro, utilizou-se a Análise de Componentes Principais (ACP) para gerar componentes principais a partir dos indicadores econômico-financeiros. Em seguida, rodou-se uma regressão logit tendo como variáveis dependentes as informações estruturais e os componentes principais mais relevantes.

\footnotetext{
2 Ver, por exemplo, os trabalhos de Rocha (1999) e Janot (2001). No que concerne a cooperativas, foram identificados apenas três trabalhos: o de Bressan et al (2004), o de Sampaio (2006) e o de Carvalho et al (2009). 3 Conforme o Manual de Supervisão do Banco Central do Brasil, "classificam-se como 'em evidência' as instituições supervisionadas que apresentam necessidade de acompanhamento específico por parte da Supervisão, decorrente de situações que comprometem ou venham a comprometer as condições indispensáveis para o funcionamento regulamentar, tais como descumprimento dos padrões mínimos de capital, grave situação dos controles internos, crise de liquidez ou outras deficiências de natureza grave (p. 31)". O Manual da Supervisão do Banco Central do Brasil pode ser encontrado no endereço eletrônico https://www3.bcb.gov.br/gmn/visualizacao/listarDocumentosManualPublico.do?method=visualizarDocument oInicial\&itemManualId $=$ null.
} 


\subsection{ANÁLISE DE COMPONENTES PRINCIPAIS}

A Tabela 3 mostra a porcentagem da variância dos dados explicada por cada um dos 10 primeiros componentes principais gerados pela ACP. Nota-se que, a partir do oitavo componente principal, o aumento na variância explicada acumulada passa a ser pequena Além disso, apenas os sete primeiros componentes principais são superiores a um. Assim, optou-se por considerar apenas os sete primeiros componentes principais, que explicam uma porcentagem significativa (quase $82 \%$ ) da variância dos dados. A Tabela 4 traz os autovetores para os sete primeiros componentes principais, o que permite avaliar o peso de cada indicador na formação dos componentes principais.

\subsection{REGRESSÃO LOGIT E RESULTADOS}

O próximo passo foi a estimação do modelo logit. Inicialmente, todas as variáveis estruturais da Tabela 1 e os sete primeiros componentes principais foram incluídos como variáveis independentes. Em seguida, foram utilizadas como variáveis independentes apenas as que se mostraram significativas. O resultado está apresentado na Tabela 5.

A partir dos resultados apresentados na Tabela 5, é possível fazer uma análise dos elementos relacionados à insolvência de cooperativas. O CP 1 agrega os indicadores relativos à inadimplência (ver Tabela 4). Este componente possui um coeficiente positivo, sugerindo que cooperativas com elevada inadimplência possuem maiores chances de experimentarem problemas futuros.

O CP 2 representa os indicadores de capital e liquidez; aparece com coeficiente negativo: cooperativas com altos índices de capitalização e liquidez são mais saudáveis. O CP 3 representa as cooperativas com alta imobilização, alta alavancagem e baixa rentabilidade. Seu coeficiente negativo indica que cooperativas com essas características são menos vulneráveis. Uma elevada rentabilidade por parte da cooperativa pode significar i) que ela está emprestando a seus cooperados a taxas elevadas ou ii) que ela está aplicando suas sobras em aplicações de risco. Em qualquer um dos casos, isso é prejudicial à cooperativa. A combinação entre baixa alavancagem e alta rentabilidade também é indicativo de problemas, já que as duas situações são inconsistentes: cooperativas pouco alavancadas devem experimentar uma rentabilidade menor. Finalmente, o CP 4 representa cooperativas com 
baixa rentabilidade. Seu coeficiente negativo está coerente com a relação entre rentabilidade e fragilização da cooperativa discutida acima.

A concentração da carteira de crédito, expressa pela variável MDPR, também aumenta as chances da cooperativa entrar em evidência. Um elemento que aparece fortemente relacionado à saúde da cooperativa é o fato de a mesma ser filiada a alguma cooperativa central. De fato, a central complementa a fiscalização realizada pelo Banco Central, exercendo um papel fundamental na manutenção da solvabilidade de suas filiadas. As cooperativas localizadas nas Regiões Sudeste, Sul e, principalmente, Centro-Oeste mostraram menores probabilidades de entrar em evidência do que as do Nordeste. Por fim, as cooperativas de menor porte mostraram-se mais saudáveis que as de porte médio.

O modelo apresentou um bom poder de previsibilidade. Adotando-se um cut-off de 0,05, o modelo previu corretamente a futura situação das cooperativas, no caso daquelas que não entraram em evidência, em $75 \%$ dos casos. Em se tratando das que entraram em evidência, esse valor foi de $78 \%$. Esses percentuais de acerto variam conforme se altera o nível de cut-off (Tabela 6).

\section{CONSIDERAÇÕES FINAIS}

O presente trabalho desenvolveu um modelo de previsão de insolvência para cooperativas de crédito. O modelo aqui desenvolvido apontou como fatores relacionados a uma maior probabilidade de insolvência das cooperativas de crédito os seguintes elementos: alta inadimplência, baixa capitalização, baixa liquidez, alta rentabilidade (principalmente se associada à baixa alavancagem), concentração da carteira de crédito e não filiação a uma cooperativa central.

Nota-se que os determinantes de fragilização das cooperativas de crédito refletem suas características estruturais peculiares ${ }^{5}$. A principal função da cooperativa de crédito é fornecer crédito a seus associados, portanto maus indicadores de crédito (inadimplência e concentração da carteira) aparecem associados a uma maior chance de fragilização das cooperativas. Além disso, o lucro não só não faz parte do objeto social da cooperativa, como também pode indicar que empréstimos a seus associados estão sendo feitos a taxas elevadas. Assim, uma elevada rentabilidade também aumenta as chances de insolvência de uma

${ }^{4}$ Cut-off é o nível de risco acima do qual o modelo classificará a cooperativa como uma futura cooperativa em evidência. Se o nível de risco da cooperativa for igual ao inferior a esse valor, o modelo estipulará que a mesma não entrará em evidência.

${ }^{5}$ Sobre as particularidades das cooperativas de crédito vis-à-vis outras instituições financeiras, ver Meinen (2002). 
cooperativa de crédito. Reforça-se a ideia, portanto, de que os elementos relacionados à sobrevivência das cooperativas de crédito merecem uma análise separada.

\section{TABELAS}

TABELA 1 - VARIÁVEIS ESTRUTURAIS

\begin{tabular}{lr}
\hline \multicolumn{1}{c}{ Variável } & \multicolumn{1}{c}{ Descrição } \\
\hline MDPR & 1 se (Percentual maior devedor CA)/PR $>10 \%$ \\
RURAL* & 1 se do tipo crédito rural \\
LADM & 1 se do tipo livre admissão ou Luzzatti \\
MUTUO & 1 se do tipo crédito mútuo \\
FILIADA & 1 se filiada \\
SE & 1 se da Região Sudeste \\
SUL & 1 se sediada na Região Sul \\
NORTE & 1 se sediada na Região Norte \\
NE* & 1 se sediada na Região Nordeste \\
CO & 1 se sediada na Região Centro-Oeste \\
GDE & 1 se de porte grande \\
MEDIO* & 1 se de porte médio \\
PEQUENO & 1 se de porte pequeno \\
MICRO & 1 se de porte micro \\
\hline
\end{tabular}

$(*)$ : Grupo de referência na regressão logit.

TABELA 2 - INDICADORES ECONÔMICO-FINANCEIROS

\begin{tabular}{|c|c|}
\hline Sigla & Nome \\
\hline C_INAD_15 & Inadimplência acima de 15 dias \\
\hline C_INAD_60 & Inadimplência acima de 60 dias \\
\hline C_INAD_90 & Inadimplência acima de 90 dias \\
\hline I_A_3_0_00_0 & Participação de Ativos de Renda de Intermediação Financeira no Ativo Total Ajustado \\
\hline I_A_3_1_00_0 & Operações Vencidas em relação à Carteira Total Classificada \\
\hline I_A_3_1_01_0 & Cobertura de Operações de Crédito Vencidas \\
\hline I_A_3_1_04_0 & Carteira Classificada do nível D a H \\
\hline I_C_3_1_01_0 & Adequação de Capital nível 1 \\
\hline I_C_3_1_02_0 & Adequação de Capital nível 1 e 2 (Índice de Basiléia) \\
\hline I_C_3_1_03_0 & Índice de Imobilização \\
\hline I_C_3_2_01_0 & Alavancagem \\
\hline I_D_1_1_00_0 & Custo Operacional \\
\hline I_D_1_3_00_0 & Cobertura de Despesas Administrativas com Serviços \\
\hline I_E_1_2_00_0 & Taxa de Crescimento do Patrimônio Líquido Ajustado \\
\hline I_L_1_2_00_0 & Caixa Livre em relação ao Ativo Total Ajustado \\
\hline I_L_1_4_00_0 & Ativos Líquidos em relação às Exigibilidades Imediatas \\
\hline I_L_1_5_00_0 & Ativos Líquidos em relação ao Ativo Total Ajustado \\
\hline I_L_1_7_01_0 & Ativos de Renda da Intermediação Financeira em relação aos Passivos Onerosos \\
\hline I_R_7_2_01_0 & Taxa de Aplicação Líquida dos Ativos de Renda da Intermediação Financeira \\
\hline I_R_7_2_03_0 & Rentabilidade dos Ativos de Renda da Intermediação Financeira - conceito de spread \\
\hline I_R_9_2_00_0 & Rentabilidade do Ativo Total Ajustado Médio \\
\hline I_R_9_3_00_0 & Rentabilidade do Patrimônio Líquido Ajustado Médio \\
\hline I_R_9_6_04_0 & Ponto de Equilíbrio (Margem Líquida de Intermediação Mínima) \\
\hline
\end{tabular}


TABELA 3 - AUTOVALORES.

\begin{tabular}{|c|c|c|c|c|c|c|c|}
\hline \multicolumn{2}{|c|}{ Componente Principal } & \multicolumn{2}{|c|}{ Autovalor } & \multicolumn{2}{|c|}{ Variância explicada } & \multicolumn{2}{|c|}{ Var. explicada acumulada } \\
\hline 1 & & & 5,440 & & 23,65 & & 23,65 \\
\hline 2 & & & 4,470 & & 19,43 & & 43,09 \\
\hline 3 & & & 2,734 & & 11,88 & & 54,97 \\
\hline 4 & & & 2,149 & & 9,34 & & 64,31 \\
\hline 5 & & & 1,500 & & 6,52 & & 70,83 \\
\hline 6 & & & 1,298 & & 5,64 & & 76,48 \\
\hline 7 & & & 1,211 & & 5,27 & & 81,74 \\
\hline 8 & & & 0,943 & & 4,10 & & 85,84 \\
\hline 9 & & & 0,872 & & 3,79 & & 89,63 \\
\hline 10 & & & 0,513 & & 2,23 & & 91,86 \\
\hline \multicolumn{8}{|c|}{ TABELA 4 - AUTOVETORES } \\
\hline Indicador & CP01 & CP02 & CP03 & CP04 & CP05 & CP06 & CP07 \\
\hline C_INAD_15 & 0,3727 & 0,1067 & $-0,1842$ & $-0,0115$ & 0,1038 & 0,1598 & $-0,0248$ \\
\hline C_INAD_60 & 0,3778 & 0,1190 & $-0,1698$ & 0,0235 & 0,0581 & 0,1566 & 0,0519 \\
\hline C_INAD_90 & 0,3721 & 0,1155 & $-0,1611$ & 0,0380 & 0,0464 & 0,1522 & 0,0637 \\
\hline I_A_3_0_00_0 & $-0,1708$ & $-0,2182$ & $-0,0884$ & 0,1760 & $-0,0522$ & 0,3821 & $-0,0708$ \\
\hline I_A_3_1_00_0 & 0,3730 & 0,1067 & $-0,1827$ & $-0,0096$ & 0,1025 & 0,1584 & $-0,0274$ \\
\hline I_A_3_1_01_0 & 0,0124 & $-0,0524$ & 0,1278 & 0,0726 & $-0,2640$ & $-0,3808$ & 0,6225 \\
\hline I_A_3_1_04_0 & 0,3246 & 0,0787 & $-0,0719$ & 0,0729 & $-0,0065$ & $-0,0635$ & 0,3743 \\
\hline I_C_3_1_01_0 & $-0,0644$ & 0,3629 & 0,1959 & 0,2561 & 0,1914 & $-0,0142$ & 0,0173 \\
\hline I_C_3_1_02_0 & $-0,0641$ & 0,3631 & 0,1952 & 0,2568 & 0,1923 & $-0,0116$ & 0,0179 \\
\hline I_C_3_1_03_0 & 0,1197 & $-0,2126$ & 0,3990 & $-0,2337$ & 0,0881 & 0,2565 & 0,0684 \\
\hline I_C_3_2_01_0 & 0,0929 & $-0,1554$ & 0,4383 & $-0,1904$ & 0,1138 & 0,3352 & 0,0318 \\
\hline I_D_1_1_00_0 & 0,2907 & $-0,0293$ & 0,1366 & 0,1756 & $-0,1658$ & $-0,3040$ & $-0,2196$ \\
\hline I_D_1_3_00_0 & 0,0218 & $-0,1233$ & $-0,0888$ & $-0,0460$ & 0,0601 & $-0,0549$ & 0,3781 \\
\hline I_E_1_2_00_0 & $-0,0097$ & $-0,0610$ & $-0,0578$ & 0,0396 & 0,1478 & $-0,2825$ & $-0,3302$ \\
\hline I_L_1_2_00_0 & 0,0061 & $-0,2614$ & 0,0259 & 0,1492 & 0,5560 & $-0,1567$ & 0,0912 \\
\hline I_L_1_4_00_0 & $-0,0825$ & 0,3018 & 0,2058 & 0,2569 & 0,0232 & 0,0812 & 0,0523 \\
\hline I_L_1_5_00_0 & 0,0111 & $-0,2434$ & 0,0187 & 0,2343 & 0,5739 & $-0,1128$ & 0,0769 \\
\hline I_L_1_7_01_0 & $-0,0623$ & 0,3768 & 0,2118 & 0,1557 & $-0,0230$ & 0,1534 & 0,0142 \\
\hline I_R_7_2_01_0 & $-0,0705$ & 0,2058 & $-0,0488$ & $-0,5347$ & 0,2186 & $-0,1093$ & 0,0738 \\
\hline I_R_7_2_03_0 & $-0,1022$ & 0,2878 & 0,0176 & $-0,4455$ & 0,2179 & $-0,0725$ & 0,0787 \\
\hline I_R_9_2_00_0 & $-0,2828$ & 0,1633 & $-0,2201$ & $-0,0501$ & 0,0983 & 0,1795 & 0,2096 \\
\hline I_R_9_3_00_0 & $-0,1952$ & 0,0969 & $-0,4622$ & 0,0305 & 0,0904 & $-0,0579$ & $-0,0675$ \\
\hline I_R_9_6_04_0 & 0,2132 & 0,1368 & 0,1946 & $-0,2098$ & 0,0284 & $-0,3642$ & $-0,2747$ \\
\hline
\end{tabular}

Obs.: Os indicadores mais relevantes na formação de cada componente principal estão em negrito. 


\begin{tabular}{|c|c|c|c|}
\hline \multicolumn{4}{|c|}{ TABELA 5 - REGRESSÃO LOGIT } \\
\hline Variável & Coeficiente & Erro-padrão & Probabilidade \\
\hline СР01 & 0,2370 & 0,0507 & 0,0000 \\
\hline СР02 & $-0,2388$ & 0,1084 & 0,0277 \\
\hline СР03 & $-0,2641$ & 0,0765 & 0,0006 \\
\hline СР04 & $-0,4742$ & 0,0996 & 0,0000 \\
\hline MDPR & 1,1973 & 0,3175 & 0,0002 \\
\hline FILIADA & $-2,2227$ & 0,3254 & 0,0000 \\
\hline SE & $-0,9982$ & 0,2742 & 0,0003 \\
\hline SUL & $-2,4204$ & 0,4738 & 0,0000 \\
\hline $\mathrm{CO}$ & $-2,8099$ & 0,8672 & 0,0012 \\
\hline PEQUENO & $-1,3833$ & 0,3280 & 0,0000 \\
\hline MICRO & $-2,1922$ & 0,4887 & 0,0000 \\
\hline \multicolumn{4}{|c|}{ TABELA 6 - PORCENTAGEM DE ACERTO DO MODELO } \\
\hline Cut-off & EVID $=0$ ( 1.135 cooperativas $)$ & $E V I D=1$ (54 cooperativas) & Total \\
\hline 0,03 & 64,67 & & 65,77 \\
\hline 0,04 & 68,99 & & 69,64 \\
\hline 0,05 & 74,98 & & 75,11 \\
\hline 0,06 & 80,35 & & 79,98 \\
\hline 0,07 & 84,32 & & 83,60 \\
\hline
\end{tabular}

\section{REFERÊNCIAS}

BRESSAN, V. G. F., M. J. BRAGA \& J. E. DE LIMA. Análise de Insolvência das Cooperativas de Crédito Rural do Estado de Minas Gerais. Estudos Econômicos, v. 34, n. 3, p. 553-585, 2004.

ENCONTRO NACIONAL DE ECONOMIA, 37, 2009, Foz do Iguaçu. Fatores Relacionados à Mortalidade e Longevidade de Cooperativas de Crédito Brasileiras. Foz do Iguaçu: 2009.

JANOT, M.. Modelos de previsão de insolvência bancária no Brasil. Trabalhos para Discussão, n. 13. Brasília: Banco Central do Brasil, 2001.

MEINEN, E.. Cooperativismo de Crédito: raízes, evolução e particularidades. In: DOMINGUES, J. A. S.. Cooperativas de Crédito no Direito Brasileiro. Porto Alegre: Sagra Luzzatto, 2002. p. 11-20.

PINHEIRO, M. A. H. Cooperativas de Crédito: História da Evolução Normativa no Brasil. 6. e5d. Brasília: Banco Central do Brasil, 2008.

ROCHA, F.. Previsão de falência bancaria: um modelo de risco proporcional. Pesquisa e Planejamento Econômico, v. 29, n. 1, p. 137-152, 1999.

SAMPAIO, J. N. de P.. Modelo de Previsão de Insolvência de Cooperativas de Crédito Mútuo Urbanas. 72 f. Dissertação (Mestrado) - Universidade Federal do Ceará, 2006.

SOARES, M. M. \& A. D. DE MELO SOBRINHO. Microfinanças: O Papel do Banco Central do Brasil e a Importância do Cooperativismo de Crédito. 2. ed. Brasília: Banco Central do Brasil, 2008.

WORLD COUNCIL OF CREDIT UNIONS - WOCCU. Statistical Report. 2008. 
